\title{
Soluble urokinase-type plasminogen activator receptor is a novel biomarker predicting acute exacerbation in COPD
}

This article was published in the following Dove Press journal:

International Journal of COPD

13 February 2015

Number of times this article has been viewed

\section{Aziz Gumus' \\ Nejat Altintas ${ }^{2}$ \\ Halit Cinarka' \\ Aynur Kirbas $^{3}$ \\ Muge Hazıroglu' \\ Mevlut Karatas' \\ Unal Sahin'}

'Department of Pulmonary Medicine, School of Medicine, Recep Tayyip Erdogan University, Rize, Turkey; ${ }^{2}$ Department of Pulmonary

Medicine, School of Medicine, Namik Kemal University, Tekirdag, Turkey; ${ }^{3}$ Department of Clinical Biochemistry, School of Medicine, Recep Tayyip Erdogan University, Rize, Turkey
Correspondence: Nejat Altintas Department of Pulmonary Medicine, School of Medicine, Namik Kemal University, 100 Yil Mah, Tunca Caddesi,

Tekirdag, Turkey

Tel +902822500000

Email nejataltintas@gmail.com
Background: Chronic obstructive pulmonary disease (COPD) is a chronic inflammatory condition, and progresses with acute exacerbations. (AE). During AE, levels of acute phase reactants such as C-reactive protein (CRP) and inflammatory cells in the circulation increase. Soluble urokinase-type plasminogen activator receptor (suPAR) levels increase in acute viral and bacterial infections and in diseases involving chronic inflammation. The purpose of this study was to investigate the effectiveness of suPAR in predicting diagnosis of AE of COPD (AE-COPD) and response to treatment.

Methods: The study population consisted of 43 patients diagnosed with AE-COPD and 30 healthy controls. suPAR, CRP, and fibrinogen levels were measured on the first day of hospitalization and on the seventh day of treatment.

Results: We found that fibrinogen $(P<0.001)$, CRP $(P<0.001)$, and suPAR $(P<0.001)$ were significantly higher in patients with AE-COPD than in healthy controls. Fibrinogen $(P<0.001)$, CRP $(P=0.001)$, and suPAR $(P<0.001)$ were significantly decreased by the seventh day of treatment. However, the area under receiver operator characteristic curve showed that suPAR is superior to CRP and fibrinogen in distinguishing AE-COPD. There was a correlation between fibrinogen, CRP, and suPAR. However, only fibrinogen was a powerful predictor of suPAR in multiple linear regression. In multiple logistic regression, only suPAR and fibrinogen were strong predictors of AE-COPD ( $P=0.002$ and $P=0.014$, respectively). Serum suPAR was negatively correlated with forced expiratory volume in 1 second $(r=-478, P=0.001)$.

Conclusion: suPAR is a marker of acute inflammation. It is well correlated with such inflammation markers as CRP and fibrinogen. suPAR can be used as a predictor of AE-COPD and in monitoring response to treatment.

Keywords: biomarker, chronic obstructive pulmonary disease, inflammation, suPAR

\section{Introduction}

Chronic obstructive pulmonary disease (COPD) is characterized by recurrent episodes of exacerbations defined by an acute worsening in the severity of a patient's symptoms, including baseline dyspnea and coughing, that warrants a change in medication or medical intervention. ${ }^{1}$ Exacerbations have been linked to disease-associated morbidity and mortality, placing significant strain on medical facilities, increasing resource burden, and driving up ongoing health care costs. ${ }^{2}$

The importance of exacerbation prediction has recently been recognized in the application of effective and aggressive prevention strategies. ${ }^{1}$ While major advances have been made in the past decade, measurable prognostic factors predicting exacerbation risk in COPD patients remain undefined, which is partly due to controversies in the definition 
of acute exacerbation of COPD (AE-COPD): ${ }^{3}$ whether the definition depends solely on symptoms or health care usage, diagnostic criteria remain subjective. This generates difficulty in objectively differentiating exacerbations from day-to day variations. Therefore, a "biomarker" is needed that can yield an objective verification of exacerbations. In addition, a good biomarker may not only indicate the exacerbations, but may also predict the severity of exacerbations. ${ }^{4}$

Urokinase-type plasminogen activator receptor (UPAR) is expressed on various cell types, and the complex interaction with its ligand urokinase plasminogen activator (uPA) has been shown to promote tissue invasion in malignant diseases by converting plasminogen into plasmin, resulting in degradation of the extracellular matrix., ${ }^{5,6}$ Migration of inflammatory cells from the bloodstream into tissues is an essential component of inflammation and the immune response against infection in which the UPAR/uPA system is directly involved. ${ }^{7}$ During inflammatory stimulation, uPAR is cleaved from the cell surface by proteases to create the soluble form of the receptor, suPAR, which can be detected in blood, urine, and cerebrospinal fluid. ${ }^{8}$ High suPAR levels were shown to be associated with an undesirable outcome in HIV infection, ${ }^{9}$ active pulmonary tuberculosis, ${ }^{10}$ bacterial meningitis, ${ }^{8}$ and pneumococcal bacteremia, ${ }^{11}$ suggesting that it may be a good biomarker of inflammation. ${ }^{12}$

In recent years, there have been some studies investigating the potential role of biomarkers during the inflammatory process of chronic diseases. ${ }^{13,14}$ However, to our knowledge, the effectiveness of the inflammation marker, suPAR, in predicting AE-COPD and in response to treatment has not been investigated so far. We hypothesized that serum suPAR would be increased in patients with AE-COPD, and that it would be decreased by treatment. The study also examined how serum suPAR levels changed with degree of airflow obstruction and with serum level of fibrinogen and CRP.

\section{Materials and methods}

\section{Study population}

In this cross-sectional study, consecutive patients with AE-COPD were prospectively recruited at Recep Tayyip Erdogan University Hospital, Rize, Turkey between January 2013 and April 2014. The disease control group comprised subjects (both nonsmokers and smokers) who had no history of pulmonary disease.

\section{Study design}

The primary objective of the study was to test the hypothesis that suPAR is a blood biomarker, the level of which rises during AE-COPD and decreases with 7 days of treatment.
The secondary objective of the study was to show a relationship between suPAR and other putative biomarkers: CRP and fibrinogen.

Smoking status, history of cardiovascular and respiratory disease, diabetes mellitus, and use of medications were documented with help of a questionnaire. Patients with AECOPD were treated for 7 days and reevaluated to observe the changes in suPAR levels in response to treatment.

\section{Exclusion criteria}

Patients who had conditions that could potentially affect circulating suPAR levels were excluded. Accordingly, patients previously diagnosed with bronchial asthma, bronchiectasis, cystic fibrosis, upper airway obstruction, or bronchiolitis related to systemic pathology; patients requiring monitoring in the intensive care unit due to exacerbation; and patients with active cancer, cirrhosis of the liver, acute or chronic kidney failure, heart failure, uncontrolled hypertension, and uncontrolled diabetes mellitus were excluded. ${ }^{12}$

\section{Diagnosis of COPD}

A diagnosis of COPD was made according to the criteria of GOLD, as follows: the diagnosis of COPD was based on current or past smoking history ( $>20$ pack-years), clinical evaluation, and pulmonary function testing showing airflow obstruction (forced expiratory volume in 1 second $\left[\mathrm{FEV}_{1}\right] /$ forced vital capacity ratio of $<0.7$ ) with a change in $\mathrm{FEV}_{1}$ of less than $200 \mathrm{~mL}$ and $12 \%$ in the bronchodilator test. ${ }^{15}$

\section{Definition of AE-COPD}

Acute exacerbation was defined as prolonged ( $\geq 48$ hours) worsening of dyspnea sensation, coughing, or sputum production that can become purulent leading to an increase in the use of maintenance medications and/or supplementation with additional medications. ${ }^{16}$

\section{Pulmonary function tests}

Pulmonary function tests were performed at least three times with a ZAN system (ZAN ${ }^{100}$; ZAN Gerätetechnik GmbH, Oberthulba, Germany) to all COPD patients when the patients were in a stable period. Staging of airflow limitation was made according to GOLD guidelines (GOLD stage I $\left[\mathrm{FEV}_{1} \geq 80 \%\right]$, stage II $\left[50 \% \leq \mathrm{FEV}_{1}<80 \%\right.$ ], stage III $\left[30 \% \leq \mathrm{FEV}_{1}<50 \%\right]$, and stage IV $\left.\left[\mathrm{FEV}_{1}<30 \%\right]\right) .{ }^{15}$

\section{Serum suPAR measurement}

Blood samples were collected upon admission to the hospital (prior to therapeutic interventions) as well as in the morning 
of day 7 after admission. After centrifugation at $2,000 \times g$ at $4^{\circ} \mathrm{C}$ for 10 minutes, serum and plasma aliquots of $1 \mathrm{~mL}$ were frozen immediately at $-80^{\circ} \mathrm{C}$.

Serum levels of suPAR were measured quantitatively using the enzyme-linked immunosorbent assay with the commercially available suPARnostic kit ${ }^{\circledR}$, according to the manufacturer's instructions (ViroGates, Copenhagen, Denmark). The technicians who measured the samples were blinded to the identity of the patient samples.

\section{Ethical concerns}

All participants provided written informed consent. The study was approved by the local ethical committee (number $=2012 / 134$ ) and conducted in accordance with the Declaration of Helsinki.

\section{Statistical analysis}

Results for normally distributed continuous variables are expressed as the mean value \pm standard deviation, and continuous variables with nonnormal distribution are presented as median values and interquartile range. Analyses of normality in the continuous variables were performed using the Shapiro-Wilk test, histograms, and Q-Q plots. Categorical variables were expressed as numbers and percentage. The chi-square test was used to compare proportions in different groups. Student's $t$-test or Mann-Whitney $U$-test was used to compare the two independent groups according to distribution state. If tests of normality were met, one-way analysis of variance was used to compare more than two groups; the cutoff level of $\alpha$ error was reduced to $0.005 /$ (number of tests) (Bonferroni correction), and the KruskalWallis test was used when tests of normality failed. In cases where the Kruskal-Wallis test yielded a statistical significance, post hoc analysis was performed to identify the groups which showed differences by Bonferroni-corrected Mann-Whitney U-test.

Correlations between levels of circulating suPAR and CRP were determined by Pearson correlation. These two parameters were logarithmically transformed because they were not normally distributed.

Univariate associations between continuous baseline characteristics and the presence of AE-COPD were assessed with logistic regression analysis. The Wald test was used to obtain logistic regression analysis parameters. In all multivariate models, backward stepwise selection was used to derive the final model, and significance levels of 0.05 and 0.1 were chosen to include and exclude terms, respectively. Variables that correlated significantly with suPAR levels in univariate analysis (Pearson correlation) were included in a backward stepwise multiple linear regression analysis. In these models, we forced in age, sex, body mass index (BMI), arterial partial pressure of oxygen, inhaled steroid use, pack-years of smoking, hypertension, and platelet count as covariates to adjust for their potential effects on SuPAR.

The area under the curve, receiver operating characteristics (ROCs) for each marker were analyzed, to differentiate AE-COPD from the same patient group in whom specific exacerbation treatment was given for 7 days. All statistical analyses were performed with SPSS software (version 21.0; IBM Corporation, Armonk, NY, USA). A two-sided probability value less than 0.05 was considered statistically significant.

\section{Results}

This prospective study recruited 43 patients (aged $68 \pm 8$ years; 37 male; $27.4 \pm 4.6 \mathrm{~kg} / \mathrm{m}^{2}$ ) diagnosed with AE-COPD who were age-, sex-, and BMI-matched with 30 healthy controls. Patients with AE-COPD $(n=43)$ were treated for 7 days and reassessed as a newly paired group of patients with AE-COPD.

There were no differences between the COPD and the healthy control groups in relation to smoking history. Most of the patients with AE-COPD had severe airflow obstruction; $81 \%$ of the patients had FEV $1<50 \%$ predicted. There were no mild COPD cases in the study population. This was not a planned outcome. The characteristics and laboratory findings of participants in the groups and pulmonary function tests of the COPD group are outlined in Table 1.

There were no intergroup differences with respect to high-density lipoprotein (HDL) cholesterol, low-density lipoprotein (LDL) cholesterol, triglycerides, and fasting concentrations of glucose, or to the use of lipid-lowering medications (data not shown).

\section{Serum suPAR in the study groups}

Median plasma suPAR in patients with AE-COPD $(4.84 \pm 1.90 \mathrm{ng} / \mathrm{mL})$ was significantly higher than in healthy controls $(2.36 \pm 0.89 \mathrm{ng} / \mathrm{mL} ; P<0.001)$ (Figure 1). Serum suPAR levels measured on the first and seventh days were compared to evaluate the response to treatment in patients with AE-COPD. A significant decrease in median suPAR level was observed after treatment, from $4.84 \pm 1.90 \mathrm{ng} / \mathrm{mL}$ to $3.38 \pm 1.34 \mathrm{ng} / \mathrm{mL}(P<0.001)$ as shown in Figure 2 .

There was a significant inverse correlation between plasma suPAR levels and post-bronchodilator $\mathrm{FEV}_{1}(\%$ predicted) $(r=-0.478, P=0.001)$ as shown in Figure 3. Figure 3 shows suPAR data at admission and $\mathrm{FEV}_{1}$ data at the stable period of patients with AE-COPD. 
Table I Characteristics, laboratory findings, and pulmonary function tests of study groups

\begin{tabular}{|c|c|c|c|}
\hline & Healthy control $(n=30)$ & AE-COPD $(n=43)$ & $P$-value \\
\hline Age, years, mean (SD) & $64(7)$ & $68(8)$ & $0.346^{a}$ \\
\hline Sex, male $(n, \%)$ & $25(83)$ & $40(93)$ & $0.260^{\mathrm{b}}$ \\
\hline $\mathrm{BMI}, \mathrm{kg} / \mathrm{m}^{2}$, mean $(\mathrm{SD})$ & $28.9(4.8)$ & $27.4(4.6)$ & $0.410^{\mathrm{a}}$ \\
\hline Ever-smoker (n, \%) & $27(90)$ & $41(95)$ & $0.332^{\mathrm{b}}$ \\
\hline Current smoker (n, \%) & $10(33)$ & $10(23)$ & $0.748^{\mathrm{b}}$ \\
\hline Cigarettes (pack-years) & $40(17)$ & $53(20)$ & $0.059^{c}$ \\
\hline COPD duration (years), mean (SD) & NA & $8.3(4.2)$ & NA \\
\hline Predicted $\mathrm{FEV}, \%$ & NA & $37(12)$ & NA \\
\hline Predicted $\mathrm{FEV} / \mathrm{FVC}$ & NA & $51(10)$ & NA \\
\hline \multicolumn{4}{|l|}{ Stage of disease $(n, \%)$} \\
\hline I & NA & $0(0)$ & NA \\
\hline II & & $8(19)$ & \\
\hline III & & $24(55)$ & \\
\hline IV & & II (26) & \\
\hline \multicolumn{4}{|l|}{ Arterial blood gases, mean (SD) } \\
\hline $\mathrm{pH}$ & NA & $7.38(0.05)$ & NA \\
\hline $\mathrm{PO}_{2}(\mathrm{mmHg})$ & & $57(8)$ & \\
\hline $\mathrm{PCO}_{2}(\mathrm{mmHg})$ & & $43(9)$ & \\
\hline Patients with inhaled corticosteroids ( $\mathrm{n}, \%)$ & NA & $43(100)$ & NA \\
\hline Patients with LABA (n, \%) & NA & $43(100)$ & NA \\
\hline
\end{tabular}

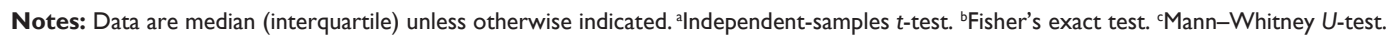

Abbreviations: AE-COPD, acute exacerbation of chronic obstructive pulmonary disease; BMI, body mass index; CRP, C-reactive protein; FEV , forced expiratory volume in I second; FVC, forced vital capacity; LABA, long-acting beta agonist; NA, not applicable; $\mathrm{PO}_{2}$, partial pressure of oxygen; $\mathrm{PCO}$, partial pressure of carbon dioxide; SD, standard deviation.

\section{Putative inflammatory markers in the study groups}

Median serum fibrinogen $(4.25 \pm 6.26 \mathrm{mg} / \mathrm{dL})$ and CRP $(577 \pm 226 \mathrm{mg} / \mathrm{dL})$ were significantly higher in patients with AE-COPD on the first day than in healthy control subjects

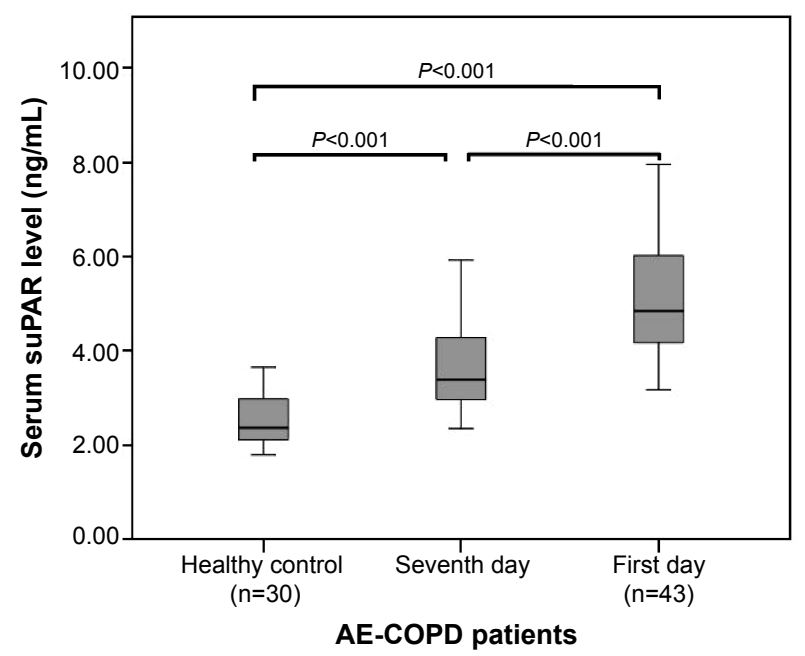

Figure I Serum soluble urokinase-type plasminogen activator receptor (suPAR) in healthy controls and in patients with acute exacerbation of chronic obstructive pulmonary disease (AE-COPD) on the first and seventh days.

Notes: Data are expressed as boxplots, in which the horizontal lines illustrate the 25 th, 50th, and 75 th percentiles of the values of suPAR. The vertical lines represent the 5 th and 95 th percentiles.
$(0.74 \pm 1.96 \mathrm{mg} / \mathrm{dL} ; P<0.001$ and $240 \pm 128 \mathrm{mg} / \mathrm{dL} ; P<0.001$, respectively) as shown in Figure 4.

Plasma concentrations of CRP and fibrinogen levels were decreased to $2.54 \pm 4.18 \mathrm{mg} / \mathrm{dL}(P<0.001)$ and $420 \pm 190 \mathrm{mg} / \mathrm{dL}(P<0.001)$, respectively, with 7 days of

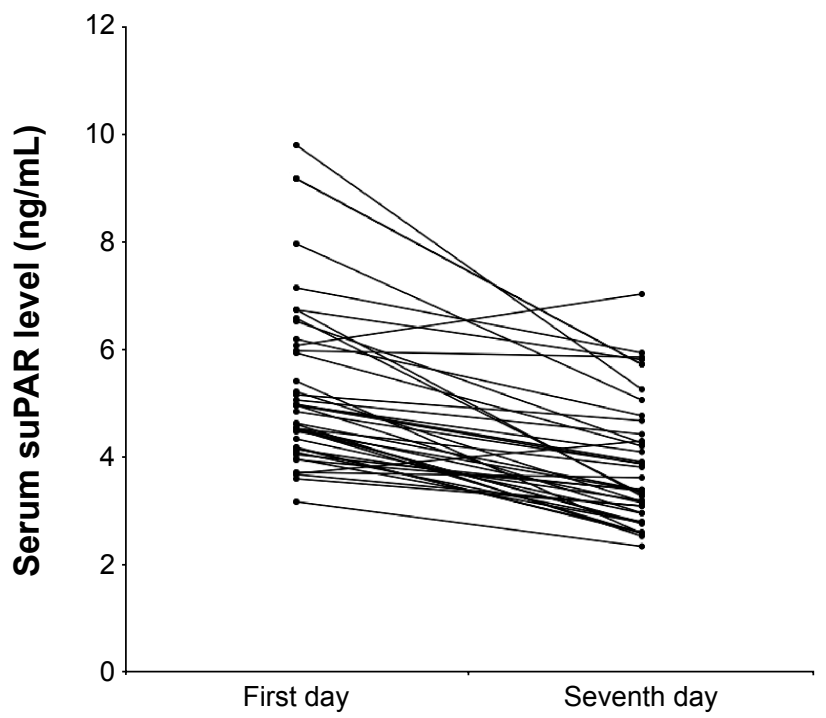

Figure 2 Serum soluble urokinase-type plasminogen activator receptor (suPAR) during an acute exacerbation and on the seventh day of treatment.

Notes: Median circulating suPAR during an acute exacerbation was $4.84 \pm 1.90 \mathrm{ng} / \mathrm{mL}$, compared to $3.38 \pm 1.34 \mathrm{ng} / \mathrm{mL}(P<0.00 \mathrm{I})$ on the seventh day of treatment. 


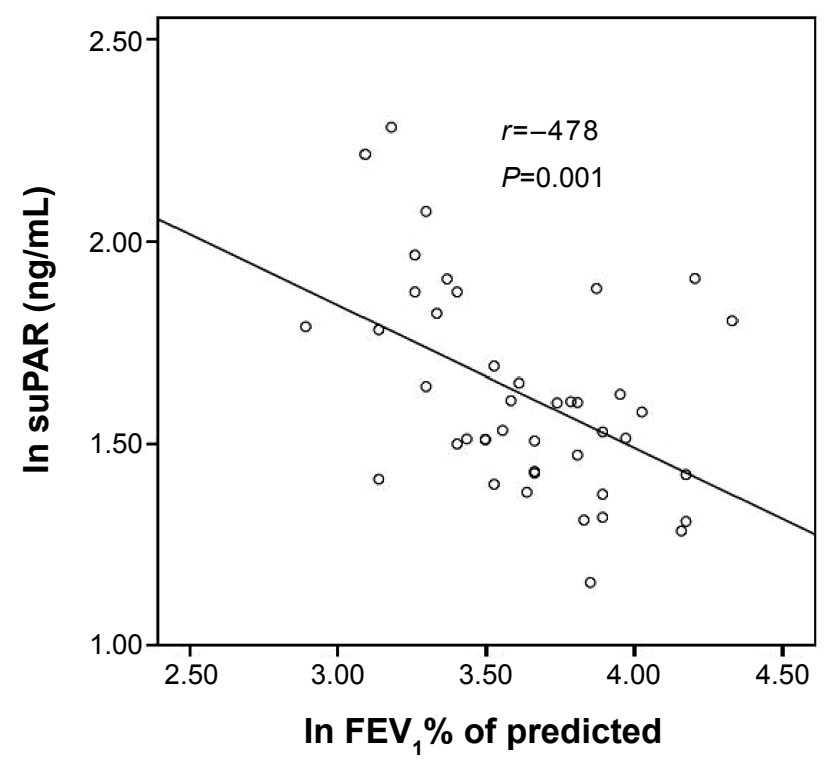

Figure 3 Serum suPAR and FEV, \% of predicted in patients with acute exacerbation of chronic obstructive pulmonary disease.

Notes: Serum suPAR was correlated inversely with $\mathrm{FEV}, \%$ of predicted using Pearson correlation $(r=-478, P=0.00 \mathrm{I})$.

Abbreviations: $\mathrm{FEV}_{1}$, forced expiratory volume in I second; In, natural logtransformed; suPAR, soluble urokinase-type plasminogen activator receptor.

treatment. CRP and fibrinogen levels on the first day and the seventh day are shown in Figure 5.

\section{Variables associated with serum suPAR}

For patients with AE-COPD on the first day, positive correlations were found between suPAR and CRP and fibrinogen

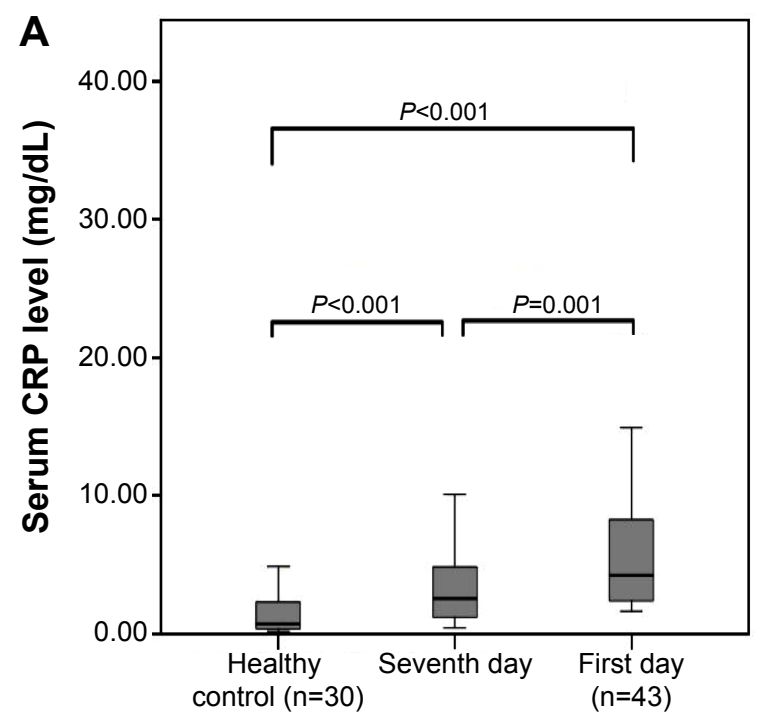

AE-COPD patients
(Table 2). Stepwise multiple regression analysis adjusting for confounding factors (age, sex, BMI, arterial partial pressure of oxygen, arterial partial pressure of carbon dioxide, $\mathrm{pH}$, pack-years of smoking, smoking status, inhaled corticosteroid therapy) revealed that only fibrinogen was a strong independent predictor $(P<0.001)$ of suPAR, explaining $58 \%$ of the total variability of circulating levels of suPAR in AE-COPD on the first day. For patients with AE-COPD on the seventh day, concentrations of suPAR also correlated positively with CRP and fibrinogen (Table 2). In stepwise multiple regression analysis, only fibrinogen was an independent predictor of suPAR ( $P=0.019)$; however, it explained only $24 \%$ of circulating levels of suPAR.

Distinguishing power of all inflammatory markers in patients with AE-COPD on the first day from the seventh day by ROC curve and logistic regression analysis

In ROC analysis, the area under the curve was $0.807(95 \%$ confidence interval $[\mathrm{CI}]: 0.715-0.900, P<0.001)$ for suPAR, 0.695 (95\% CI: 0.583-0.807, $P=0.002$ ) for CRP, and 0.763 (95\% CI: $0.663-0.862, P<0.001)$ for fibrinogen in distinguishing patients with AE-COPD on the first day from the seventh day, indicating that suPAR was superior to CRP and fibrinogen as a predictive factor in patients with AE-COPD on the first day (Figure 6).

B

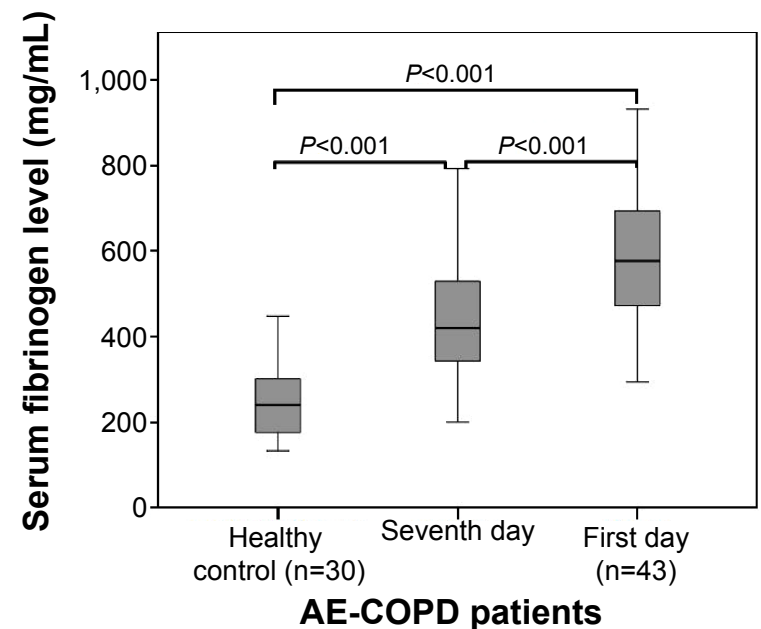

Figure 4 C-reactive protein (CRP) (A) and fibrinogen (B) in healthy subjects and patients with acute exacerbation of chronic obstructive pulmonary disease (AE-COPD) on the first and the seventh day.

Note: Box and whisker plots represent medians, interquartile ranges, and range. 

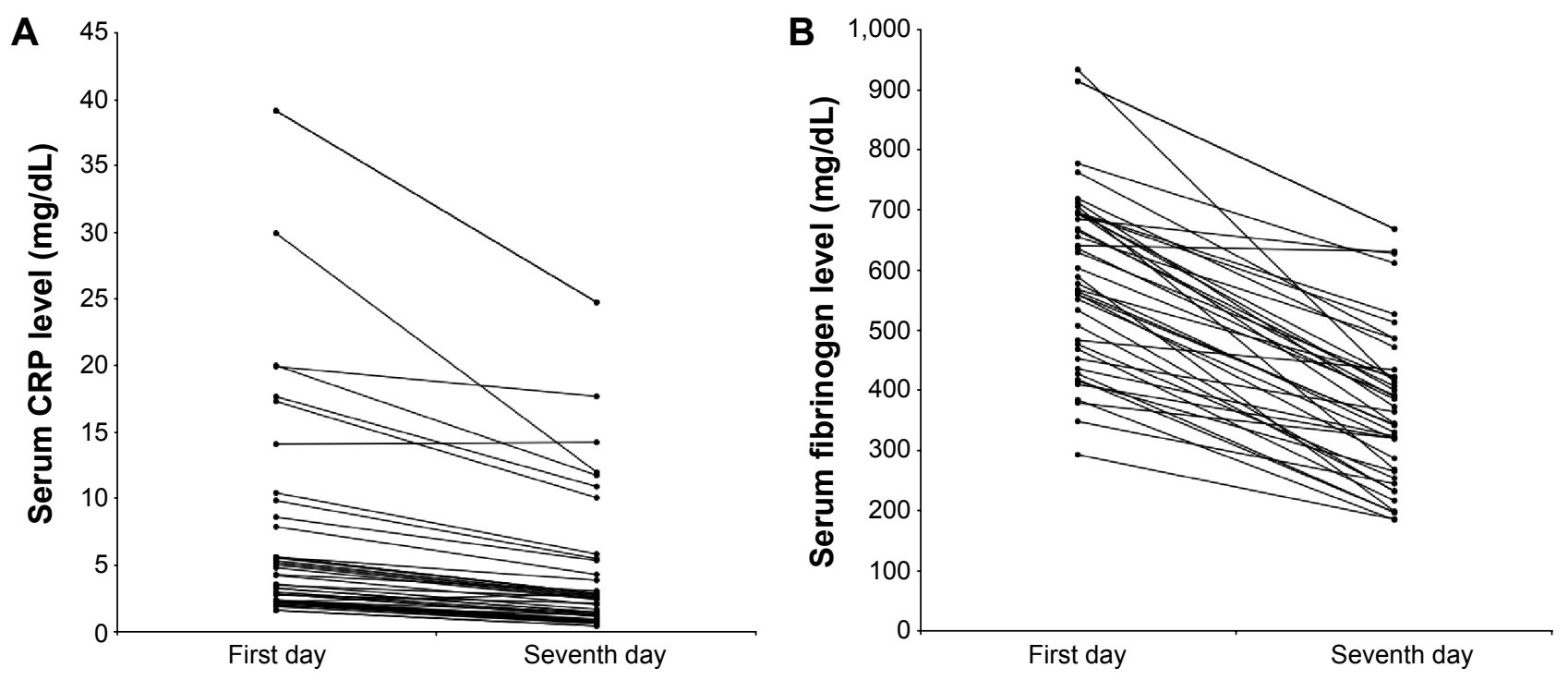

Figure 5 Serum C-reactive protein (CRP) (A) and fibrinogen (B) during an acute exacerbation and on the seventh day of the treatment.

Notes: Median circulating CRP and fibrinogen during an acute exacerbation were $4.25 \pm 6.26 \mathrm{mg} / \mathrm{dL}$ and $577 \pm 226 \mathrm{mg} / \mathrm{dL}$, compared to $2.54 \pm 4.18 \mathrm{mg} / \mathrm{dL}(P<0.00 \mathrm{I})$ and $420 \pm 190 \mathrm{mg} / \mathrm{dL}(P<0.00 \mathrm{I})$ on the seventh day of treatment, respectively.

Multiple logistic regression analysis was run to predict AE-COPD from suPAR, fibrinogen, and CRP. Only suPAR and fibrinogen were strong predictors of AE-COPD $(P=0.002$ and $P=0.014$, respectively) (Table 3 ).

\section{Discussion}

The present study uncovered the following novel findings: serum suPAR levels were significantly higher in patients with AE-COPD than in healthy controls. High serum suPAR levels were decreased with 7 days of treatment. Serum suPAR remained an independent predictor of AECOPD after multivariate adjustment. Furthermore, serum suPAR levels exhibited a positive strong correlation with the inflammatory markers CRP and fibrinogen. These findings indicate that circulating suPAR levels promise to be a useful biomarker with which to distinguish AE-COPD

Table 2 Pearson correlation coefficients of suPAR with inflammatory markers in patients with AE-COPD on the first and seventh day

\begin{tabular}{|c|c|c|c|c|}
\hline \multirow[t]{3}{*}{ Variable } & \multicolumn{4}{|c|}{$\operatorname{suPAR}^{a}(\mathrm{ng} / \mathrm{dL})$} \\
\hline & \multicolumn{4}{|c|}{ Patients with AE-COPD } \\
\hline & \multicolumn{2}{|c|}{ First day (P-value) } & \multicolumn{2}{|c|}{ Seventh day ( $P$-value $)$} \\
\hline CRPa & 0.331 & 0.030 & 0.301 & 0.049 \\
\hline Fibrinogen & 0.585 & $P<0.001$ & 0.426 & 0.004 \\
\hline Sedimentation ${ }^{\mathrm{a}}$ & 0.218 & 0.159 & 0.071 & 0.652 \\
\hline Leukocyte $^{a}$ & 0.094 & 0.549 & 0.102 & 0.514 \\
\hline
\end{tabular}

Note: ${ }^{a}$ ariables were natural log-transformed. Bold text indicates this value is statistically significant and less than $<0.05$.

Abbreviations: AE-COPD, acute exacerbation of chronic obstructive pulmonary disease; CRP, C-reactive protein; suPAR, serum soluble urokinase-type plasminogen activator receptor. and can be used to evaluate treatment response in patients with AE-COPD.

AE-COPD is a significant cause of mortality and morbidity. Therefore, diagnosis and prevention of these episodes is one of the major goals in the management of COPD. Currently, the diagnosis of an exacerbation relies exclusively on the clinical presentation of the patient complaining of an acute change of symptoms that is beyond normal day-to-day variation. ${ }^{17}$ Identification of a biomarker that has the predictive potential of identifying a forthcoming exacerbation would therefore be highly valuable. Biomarkers such as CRP and fibrinogen represent low-grade systemic inflammation, and increased levels have been found in patients with AE-COPD. ${ }^{18-20}$ However, no clear biological markers have been identified so far. ${ }^{2}$

Table 3 Univariate and multivariate logistic regression analysis of inflammatory markers in patients with acute exacerbation of chronic obstructive pulmonary disease as the dependent variable

\begin{tabular}{|c|c|c|c|c|}
\hline Analysis & $\beta$ & SE & $P$-value & OR $(95 \% \mathrm{Cl}$ for $\beta)$ \\
\hline \multicolumn{5}{|l|}{ Univariate } \\
\hline suPAR ${ }^{a}$ & || $2.84 \mid$ & 1.115 & $<\mathbf{0 . 0 0 1}$ & $|2.869-|, 003.487$ \\
\hline Fibrinogen & 1.007 & 0.002 & $<\mathbf{0 . 0 0 1}$ & $1.003-1.012$ \\
\hline CRPa & 2.229 & 0.263 & 0.002 & $1.332-3.728$ \\
\hline \multicolumn{5}{|l|}{ Multivariate } \\
\hline suPAR $^{a}$ & 64.391 & 1.316 & 0.002 & $4.882-849.342$ \\
\hline Fibrinogen & 1.005 & 0.002 & 0.014 & $1.001-1.010$ \\
\hline CRPa & 1.022 & $0.06 \mathrm{I}$ & 0.720 & $0.907-1.15 \mid$ \\
\hline
\end{tabular}

Note: ${ }^{\vee}$ ariable was natural transformed. Bold text indicates this value is statistically significant and less than $<0.05$.

Abbreviations: $\mathrm{Cl}$, confidence interval; $\mathrm{CRP}, \mathrm{C}$-reactive protein; $\mathrm{OR}$, odds ratio; suPAR, soluble urokinase-type plasminogen activator recepto; SE, standard error. 


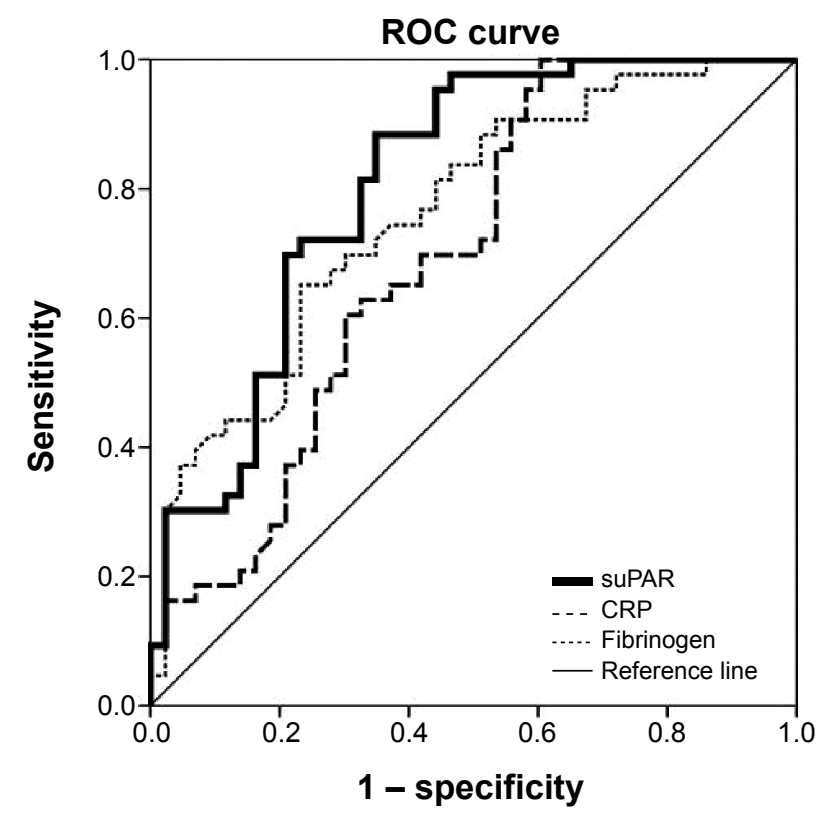

Figure 6 ROC curve for suPAR, CRP, and fibrinogen in discrimination of patients with acute exacerbation of chronic obstructive pulmonary disease on the first and seventh day.

Abbreviations: CRP, C-reactive protein; ROC, receiver operating characteristic; suPAR, soluble urokinase-type plasminogen activator receptor.

In contrast to many inflammatory markers, suPAR can potentially be a robust and independent biomarker of inflammation because suPAR is a very stable molecule and its serum concentrations are not affected by circadian changes. ${ }^{21}$ It was also shown that treatment of infectious diseases and cancer resulted in a decrease in serum concentrations of suPAR; furthermore, suPAR levels were normalized after full recovery. ${ }^{10,22}$ To verify whether suPAR is a potential biomarker of AE-COPD, we recruited the patients with $\mathrm{AE}$ COPD, and suPAR levels were measured upon admission of patients and 7 days thereafter. Our study showed that there was a significant decrease in serum suPAR levels measured on the seventh day of treatment compared to those on the first day and suPAR levels in COPD patients were higher than in the healthy controls, suggesting that suPAR might have a role as a distinguishing biomarker of AE-COPD and can be used in the assessment of treatment response.

Cross-sectional studies showed that systemic inflammatory markers are inversely related to lung function. ${ }^{1,23,24}$ Our study, consistent with previous studies, showed that serum suPAR levels were inversely associated with the degree of airflow obstruction as measured by post-bronchodilator $\mathrm{FEV}_{1}(\%$ predicted). This finding also potentiates suPAR as an inflammatory marker.

We also measured two other putative inflammatory markers, even though serum suPAR was the main objective of this study. Indeed, CRP and fibrinogen levels were found to be significantly raised in COPD subjects compared to control subjects in previous studies. ${ }^{2,25-27}$ Data from the 1,793 individuals from the Evaluation of COPD Longitudinally to Identify Predictive Surrogate Endpoints (ECLIPSE) cohort found elevated levels of CRP and fibrinogen count to be associated with the occurrence of exacerbations in the first year of follow-up. ${ }^{1,4}$ Increased CRP and fibrinogen have also been associated with increased risk for COPD hospitalizations. ${ }^{3,28,29}$ Our study showed that fibrinogen and CRP were increased in AE-COPD patients relative to healthy subjects; this finding is consistent with former studies. Our study also showed a decrease in CRP and fibrinogen levels with 7 days of treatment in patients with AE-COPD. However, the area under the ROC curve analysis showed that suPAR was superior to CRP and fibrinogen in identifying patients with AE-COPD on the first day from the seventh day. In the present study, serum suPAR levels exhibited a positive correlation with CRP and fibrinogen levels at admission of patients with AE-COPD, which is consistent with previous findings. ${ }^{4,30}$ This correlation was also maintained in parallel with reduction after 7 days of treatment. Furthermore, multilinear regression analysis revealed that only fibrinogen was a strong independent predictor of suPAR, explaining $58 \%$ of the total variability of circulating levels of suPAR. All those previous studies and our findings are powerful support for the idea that suPAR is a marker of acute inflammation and can be used in treatment response.

\section{Limitations}

There were some limitations in this study. First, this was a retrospective study and was subject to bias. Second, we used a cross-sectional design for this study, which is not the best design for investigating any causal relationships. Although we performed adjustment for significant clinical variables, there remains the possibility of residual confounding from unmeasured variables. Third, our study was not designed to elucidate the mechanistic pathways that lead to higher suPAR ratio in patients with COPD. Further studies should focus on providing a more complete understanding of the biochemical properties and regulatory mechanisms of suPAR in this patient population to be able to better evaluate changes in response to exacerbation-specific therapies. Despite the limitations of the study, we observed that individuals with AE-COPD are significantly more likely to have higher levels of suPAR, which seems a reasonable measure with which to detect exacerbations. 


\section{Conclusion}

This study showed that serum suPAR was increased in patients with AE-COPD and decreased after 7 days of treatment. This study indicates that elevated suPAR levels have potential value as a robust and independent biomarker of exacerbation in COPD and can be used in response to treatment. Prospective long-term studies are necessary to show whether suPAR levels increase further with disease progression and whether suPAR has a capacity to show comorbidities and mortality in COPD.

\section{Acknowledgments}

The study was performed at Recep Tayyip Erdogan University Hospital, Rize, Turkey. The study was approved by the local ethical committee of Recep Tayyip Erdogan University. (number $=2012 / 134$ ).

\section{Author contributions}

All authors were involved with conception and design, acquisition of data, or analysis and interpretation of data; drafting and revision of the article; final approval of the version to be published; and agree to be accountable for all aspects of the work in ensuring that questions related to the accuracy or integrity of any part of the work are appropriately investigated and resolved.

\section{Disclosure}

This is not an industry-supported study. The authors report no conflicts of interest in this work.

\section{References}

1. Vestbo J, Hurd SS, Agustí AG, et al. Global strategy for the diagnosis, management, and prevention of chronic obstructive pulmonary disease: GOLD executive summary. Am J Respir Crit Care Med. 2013;187:347-365.

2. Decramer M, Janssens W, Miravitlles M. Chronic obstructive pulmonary disease. Lancet. 2012;379:1341-1351.

3. Rodriguez-Roisin R. Toward a consensus definition for COPD exacerbations. Chest. 2000;117:398S-401S.

4. Hurst JR, Vestbo J, Anzueto A, et al; Evaluation of COPD Longitudinally to Identify Predictive Surrogate Endpoints (ECLIPSE) Investigators. Susceptibility to exacerbation in chronic obstructive pulmonary disease. N Engl J Med. 2010;363:1128-1138.

5. Ostergaard C, Benfield T, Lundgren JD, Eugen-Olsen J. Soluble urokinase receptor is elevated in cerebrospinal fluid from patients with purulent meningitis and is associated with fatal outcome. Scand J Infect Dis. 2004;36:14-19.

6. Madsen CD, Sidenius N. The interaction between urokinase receptor and vitronectin in cell adhesion and signalling. Eur J Cell Biol. 2008; 87:617-629.

7. Portelli MA, Siedlinski M, Stewart CE, et al. Genome-wide protein QTL mapping identifies human plasma kallikrein as a post-translational regulator of serum uPAR levels. FASEB J. 2014;28:923-934.

8. Thunø M, Macho B, Eugen-Olsen J. suPAR: the molecular crystal ball. Dis Markers. 2009;27:157-172.
9. Lawn SD, Myer L, Bangani N, Vogt M, Wood R. Plasma levels of soluble urokinase-type plasminogen activator receptor (suPAR) and early mortality risk among patients enrolling for antiretroviral treatment in South Africa. BMC Infect Dis. 2007;7:41.

10. Eugen-Olsen J, Gustafson P, Sidenius N, et al. The serum level of soluble urokinase receptor is elevated in tuberculosis patients and predicts mortality during treatment: a community study from GuineaBissau. Int J Tuberc Lung Dis. 2002;6:686-692.

11. Wittenhagen P, Kronborg G, Weis N, et al. The plasma level of soluble urokinase receptor is elevated in patients with Streptococcus pneumoniae bacteraemia and predicts mortality. Clin Microbiol Infect. 2004;10:409-415.

12. Eugen-Olsen J, Andersen O, Linneberg A, et al. Circulating soluble urokinase plasminogen activator receptor predicts cancer, cardiovascular disease, diabetes and mortality in the general population. J Intern Med. 2010;268:296-308.

13. Bozinovski S, Hutchinson A, Thompson M, et al. Serum amyloid a is a biomarker of acute exacerbations of chronic obstructive pulmonary disease. Am J Respir Crit Care Med. 2008;177:269-278.

14. Smith DJ, Yerkovich ST, Towers MA, Carroll ML, Thomas R, Upham JW. Reduced soluble receptor for advanced glycation end-products in COPD. Eur Respir J. 2011;37:516-522.

15. Global Strategy for the Diagnosis, Management, and Prevention of Chronic Obstructive Lung Disease: Updated 2014. Global Initiative for Chronic Obstructive Lung Disease; 2014. Available from: http:// www.goldcopd.org/uploads/users/files/GOLD_Report_2014_Jun11. pdf. Accessed July 30, 2014.

16. Halpin DM, Decramer M, Celli B, Kesten S, Liu D, Tashkin DP. Exacerbation frequency and course of COPD. Int $J$ Chron Obstruct Pulmon Dis. 2012;7:653-661.

17. Tufvesson E, Ekberg M, Bjermer L. Inflammatory biomarkers in sputum predict COPD exacerbations. Lung. 2013;191:413-416.

18. Miniati M, Monti S, Bottai M, Cocci F, Fornai E, Lubrano V. Prognostic value of C-reactive protein in chronic obstructive pulmonary disease. Intern Emerg Med. 2011;6:423-430.

19. Thomsen M, Dahl M, Lange P, Vestbo J, Nordestgaard BG. Inflammatory biomarkers and comorbidities in chronic obstructive pulmonary disease. Am J Respir Crit Care Med. 2012;186:982-988.

20. Moberg M, Vestbo J, Martinez G, Lange P, Ringbaek T. Prognostic value of C-reactive protein, leukocytes, and vitamin $\mathrm{d}$ in severe chronic obstructive pulmonary disease. Scientific World Journal. 2014;2014:140736.

21. Kofoed K, Schneider UV, Scheel T, Andersen O, Eugen-Olsen J. Development and validation of a multiplex add-on assay for sepsis biomarkers using xMAP technology. Clin Chem. 2006;52:1284-1293.

22. Ostrowski SR, Ullum H, Goka BQ, et al. Plasma concentrations of soluble urokinase-type plasminogen activator receptor are increased in patients with malaria and are associated with a poor clinical or a fatal outcome. J Infect Dis. 2005;191:1331-1341.

23. Dahl M, Tybjaerg-Hansen A, Vestbo J, Lange P, Nordestgaard BG. Elevated plasma fibrinogen associated with reduced pulmonary function and increased risk of chronic obstructive pulmonary disease. Am J Respir Crit Care Med. 2001;164:1008-1011.

24. Thorleifsson SJ, Margretardottir OB, Gudmundsson G, et al. Chronic airflow obstruction and markers of systemic inflammation: results from the BOLD study in Iceland. Respir Med. 2009;103:1548-1553.

25. Pinto-Plata VM, Müllerova H, Toso JF, et al. C-reactive protein in patients with COPD, control smokers and non-smokers. Thorax. 2006;61:23-28.

26. Wedzicha JA, Seemungal TA, MacCallum PK, et al. Acute exacerbations of chronic obstructive pulmonary disease are accompanied by elevations of plasma fibrinogen and serum IL-6 levels. Thromb Haemost. 2000;84:210-215.

27. Garcia-Rio F, Miravitlles M, Soriano JB, et al; EPI-SCAN Steering Committee. Systemic inflammation in chronic obstructive pulmonary disease: a population-based study. Respir Res. 2010;11:63. 
28. Dahl M, Vestbo J, Lange P, Bojesen SE, Tybjaerg-Hansen A, Nordestgaard BG. C-reactive protein as a predictor of prognosis in chronic obstructive pulmonary disease. Am J Respir Crit Care Med. 2007; 175:250-255.

29. Engström G, Segelstorm N, Ekberg-Aronsson M, Nilsson PM, Lindgärde F, Löfdahl CG. Plasma markers of inflammation and incidence of hospitalisations for COPD: results from a population-based cohort study. Thorax. 2009;64:211-215.
30. Donadello K, Scolletta S, Taccone FS, et al. Soluble urokinase-type plasminogen activator receptor as a prognostic biomarker in critically ill patients. J Crit Care. 2014;29:144-149.

International Journal of COPD

\section{Publish your work in this journal}

The International Journal of COPD is an international, peer-reviewed journal of therapeutics and pharmacology focusing on concise rapid reporting of clinical studies and reviews in COPD. Special focus is given to the pathophysiological processes underlying the disease, intervention programs, patient focused education, and self management protocols.
Dovepress

This journal is indexed on PubMed Central, MedLine and CAS. The manuscript management system is completely online and includes a very quick and fair peer-review system, which is all easy to use. Visit $\mathrm{http}: / / \mathrm{www}$.dovepress.com/testimonials.php to read real quotes from published authors.

Submit your manuscript here: http://www.dovepress.com/international-journal-of-chronic-obstructive-pulmonary-disease-journal 